\title{
Cobertura de vacuna antigripal en niños de riesgo durante 2007-2008 en un centro de Atención Primaria en España
}

\author{
MM. Domínguez Cajala ${ }^{2}$ A. de Arriba Muñoz, \\ L. Escosa García , JP. García Íniguezz, M. Biosca Pàmies ${ }^{\mathrm{d}}$ N N. García Sánchez \\ oPediatra. Hospital Infantil Universitario Miguel Servet. Zaragoza. España. \\ ${ }^{口} M I R$ Pediatría. Hospital Infantil Universitario Miguel Servet. Zaragoza. España. \\ 'MIR Pediatría. Hospital Infantil Universitario La Paz. Madrid. España. \\ 'Pediatra. CS Les Borges Blanques. Lleida. España. \\ ePediatra. CS Universitario Delicias Sur. Zaragoza. España.
}

Rev Pediatr Aten Primaria. 2009; I I:399-4II

Nuria García Sánchez,ngarcias@salud.aragon.es

\section{Resumen}

Introducción: los niños con enfermedades crónicas (EC) tienen más riesgo de presentar complicaciones importantes debidas a la gripe. Aunque la vacunación rutinaria anual con vacuna trivalente inactivada está indicada en estos niños, son muy pocos los que cumplen esta recomendación. El objetivo de nuestro estudio es comprobar la cobertura de vacuna antigripal en niños con alto riesgo de complicaciones $(A R C)$ en un centro de salud urbano en España.

Pacientes y métodos: durante el otoño e invierno 2007-2008 se revisó la base de datos de las historias clínicas de los niños de 0-14 años de edad, en un centro de salud. De un total de 3.479 niños, 232 presentaban ARC. Se comprobó si habían recibido la vacuna antigripal en ese año, examinando cada historia clínica informatizada.

Resultados: la cobertura global de vacuna antigripal en niños con ARC fue 43,1\%. El 98\% de los niños vacunados recibió una vacunación completa. Según las patologías subyacentes encontradas, la cobertura vacunal fue del $44 \%$ en caso de asma, 35\% en cardiopatía congénita y $42,85 \%$ en diabetes mellitus. La cobertura de vacuna antigripal fue menor en niños de familias inmigrantes con ARC que en españoles, 35,6\% frente al 45,7\%.

Conclusiones: la cobertura de vacuna antigripal en nuestros niños con ARC es mayor que la comunicada en la bibliografía médica (10-23\%). A pesar de estos buenos resultados, se necesita aumentar la vacunación antigripal en todos los niños con EC. Los profesionales de la salud deberían diseñar estrategias para conseguir una cobertura de un 75\% como un objetivo mucho mejor.

Palabras clave: Vacunación antigripal, Programa de vacunación, Niños, Adolescente.

Los autores declaran no presentar conflictos de intereses en relación con la preparación y publicación de este artículo.

La presente versión incluye la corrección de un error en la fígura 2 de la página 405, presente en la versión inicial y en la edición en soporte papel. 
Abstract

Introduction: children with chronic medical conditions (CMC) are at high-risk of suffering from serious complications due to influenza. Although routine annual influenza immunization with trivalent inactivated vaccine is recommended in these children, few of them fulfil the recommendation. The aim of our study is to assess the rate of influenza vaccine coverage among children with CMC in a Primary Care Setting in a metropolitan area in Spain.

Patients and methods: in the 2007-2008 influenza season, the computerized database of all children aged 0-14 years in a Primary Care Setting was checked. Over 3,479 children, 232 were indentified as high-risk. The administration of influenza vaccine in these children that year was assessed checking every computerized medical record.

Results: the global influenza vaccine coverage among children with CMC was $43.1 \%$. Full immunization was received in $98 \%$ of them. Depending on the underlying medical condition, the coverage was 44\% in children with Asthma, 35\% in Congenital Heart Diseases and $42.8 \%$ in Diabetes Mellitus. Children with CMC living in immigrant families had lower rate of influenza vaccination than those in Spanish families, $35.6 \%$ versus $45.7 \%$.

Conclusions: the rate of vaccine coverage among children with CMC in our setting is higher than the one reported in medical reviews (range: 10-23\%). Despite these good results, to increase the rates of vaccination among children with CMC is needed. Health care professionals should design strategies to achieve a $75 \%$ rate of influenza vaccine coverage in high-risk children as a much better goal.

Key words: Influenza vaccines, Immunization programs, Infant, Child, Adolescent.

\section{Introducción}

La gripe es una infección vírica que produce importante morbilidad y mortalidad en países desarrollados. La mayor tasa de ataque se presenta en niños, que a su vez son una pieza importante en el inicio y expansión de las epidemias. Las complicaciones más importantes derivadas de esta enfermedad aparecen en los menores de 2 años, pacientes con condiciones médicas de base y adultos de más de 65.

Aproximadamente el 7,4-14,2\% de sujetos entre 6 meses y 17 años presentan condiciones médicas de alto riesgo para complicaciones de la gripe y son candidatos a la vacunación antigripal
(VA) anual, siendo el asma el proceso más frecuentemente encontrado ${ }^{1}$.

La carga de la enfermedad en niños con asma y otras enfermedades crónicas ha sido estudiada. El 53\% de los niños fallecidos por causas relacionadas con la gripe en 2003-2004 en EE. UU. presentaba alguna enfermedad crónica². También presentan mayor riesgo de ingreso hospitalario, que varía de 2 a 4 veces más según la edad (exceso de hospitalizaciones: 2-19). Cada año por cada 1.000 niños con enfermedades crónicas se producen unas 120-200 consultas médicas y se prescriben unas 65-140 tandas de antibióticos debidas a la gripe $^{3}$. 
Por diversas razones, entre ellas la resistencia a los antivirales, la VA es la medida más eficaz para prevenir la enfermedad y sus complicaciones, con beneficios no solo en el individuo que la recibe sino también en la comunidad. Se ha comprobado que la vacunación de escolares, tanto con vacuna trivalente inactivada como con vacuna trivalente viva atenua$\mathrm{da}$, tiene efectos beneficiosos en la comunidad disminuyendo la morbilidad y mortalidad en adultos ${ }^{4,5}$. Incluso se postula que la vacunación de escolares es más eficiente que la vacunación de ancianos y sujetos de alto riesgo ${ }^{6}$.

Diversos organismos han recomendado la VA universal de los niños a partir de los 6 meses hasta los 18 años, práctica que se inició en EE. UU. en la campaña 200820097. En Europa solo Finlandia ha iniciado la vacunación sistemática de niños entre 6 y 24 meses desde el otoño de $2007^{8}$.

En nuestro medio las indicaciones para la $V A$ se recogen en las recomendaciones del Comité Asesor de Vacunas de la Asociación Española de Pediatría (AEP) que contempla, entre otras situaciones, la vacunación anual de aquellos niños que presenten enfermedades crónicas que requieren cuidado médico continuo como son: neumopatía incluida el asma, enfermedades cardiovasculares, neuropatías crónicas, diabetes y otras metabo- lopatías, enfermedades neuromusculares, situaciones que provoquen inmunodeficiencia y aquellos niños que puedan requerir tratamiento crónico con ácido acetilsalicílico, pudiéndose administrar a partir de los 6 meses de vida. Estas normas ya estaban vigentes en la campaña 2007-20089,10.

A pesar de estas recomendaciones y de las conocidas repercusiones de la gripe en niños con enfermedades de base, la cobertura vacunal en ellos es muy baja, tanto en España como en otros países.

En el ámbito de la Pediatría de Atención Primaria es de gran interés identificar qué niños son candidatos a la VA por presentar alguna condición médica crónica y cuántos de ellos reciben realmente la vacunación, para, en caso de ser necesario, proponer líneas de mejora dirigidas a los colectivos en los que se detecte bajo cumplimiento. Este interés se mantiene e incluso se incrementa ante la presencia de una pandemia gripal.

\section{Pacientes y métodos}

Tipo de estudio: estudio transversal descriptivo. Ámbito: Centro de Salud Delicias Sur de la ciudad de Zaragoza (España) dependiente del Gobierno de Aragón.

Los pacientes objeto de estudio son los niños de 0-14 años usuarios del centro 
de salud. Entre ellos se identificaron aquellos con alguna condición médica que les situase como candidatos a recibir la vacunación antigripal en esa campaña (otoño de 2007).

Durante los meses de octubre a diciembre de 2007 se estudió la base de datos clínica del citado centro, mediante el programa $\mathrm{OMI}{ }^{\oplus}$-estadística, dado que la atención a los pacientes se realiza a través del programa médico "Oficina Médica Informatizada" (OMI -versión 6). En el referido grupo etáreo se obtuvo un listado de aquellos niños que presentaban alguno de los siguientes procesos: 1) Neumopatía crónica: asma, fibrosis quística, neumonía recurrente. 2) Cardiopatías congénitas. 3) Insuficiencia renal. 4) Diabetes mellitus. 5) Enfermedades neuromusculares. 6) Enfermedades que pudieran requerir tratamiento continuado con ácido acetilsalićlico como artritis idiopática juvenil. Se hizo una explotación de datos de los niños que presentaban los códigos diagnósticos más relevantes que englobaban estas patologías.

Para identificar a los niños con neumonía recurrente, se investigó entre aquellos que habían tenido alguna neumonía, si esta se repetía de forma que fueran más de dos en un año o tres en su curso vital.
Aunque en las situaciones que pudiera existir inmunosupresión en niños está indicada la VA, no se hizo un listado de dichos pacientes para respetar la confidencialidad de los posibles niños afectos de inmunodeficiencia adquirida por el $\mathrm{VIH}$.

Se consideró que no existía indicación para la VA en los niños con asma que no presentaron crisis de obstrucción bronquial en los dos últimos años y que no seguían tratamiento para la misma.

A partir de enero de 2008 se investigó en cada niño de riesgo si había recibido la vacuna antigripal en la campaña que finalizaba el 31 de diciembre de 2007, para lo cual se investigó cada historia clínica comprobando este hecho. Posteriormente se hizo un estudio de consolidación de datos.

En cada niño identificado de riesgo se recogieron los siguientes datos: edad, sexo, nacionalidad, condición subyacente que sienta la indicación de la vacuna antigripal, situaciones que pudieran suponer una contraindicación a la administración como alergia al huevo o efectos adversos en la vacunación en años anteriores, haber recibido o no la vacuna antigripal en la fecha de estudio y número de dosis recibidas en los vacunados por primera vez de menos de 9 años.

Se hizo un análisis descriptivo para cada una de las variables estudiadas mediante 
el cálculo de proporción y porcentajes. Se determinó la proporción y el porcentaje de niños que habían recibido la VA entre el total y para cada condición médica (asma, neumonía recurrente, otras neuropatías, diabetes mellitus, cardiopatías congénitas, enfermedades neuromusculares, artritis idiopática juvenil) y para las variables edad, sexo y nacionalidad. Dado que la variable edad se clasificó en dos categorías, menores de 6 años y mayores de 6 años, se calculó la cobertura vacunal por grupo de edad. En cuanto a la nacionalidad, se consideraron dos categorías de niños, los que viven en familias españolas y los que viven en familias inmigrantes; así, los procedentes de adopción internacional se consideraron en el primer grupo. Para estudiar la posible relación entre la cobertura vacunal y las variables cualitativas edad, sexo y nacionalidad se realizaron tablas de contingencia y determinación de chi cuadrado. Se consideró que un valor de $p<0,05$ era estadísticamente significativo. Se determinó la razón de prevalencia frente a la vacunación entre los niños de familias españolas y las de los inmigrantes, así como la fracción atribuible a este hecho.

La información se registró en una hoja Exce ${ }^{\circledR}$ y posteriormente los datos se trabajaron con el paquete estadístico SPSS ${ }^{\circledR}$, versión para Windows ${ }^{\circledR} 15.0$.

\section{Resultados}

Tras estudiar la base de datos informatizada se pudo conocer que en octubre de 2007 había 3.479 niños usuarios del centro de salud, cuya edad comprendía desde 0 hasta 14 años. De la explotación de datos según los códigos diagnósticos de los grupos de riesgo se identificaron 232 niños, es decir un 6,7\% de los usuarios, candidatos a recibir la vacunación antigripal en la campaña de ese año (figura 1).

Figura 1. Características de la población estudiada.

Número de niños de 0-14 años usuarios del centro de salud 3.479

Niños identificados de riesgo.

Candidatos a la vacunación antigripal $232(6,7 \%)$
Sin factores de riesgo para la gripe $3.247(93,3 \%)$ 
Las características de los niños con condiciones de base que les hacían susceptibles de VA pueden consultarse en la tabla I. El grupo etáreo más predominante era el de edad inferior a 6 años, 141 niños (60,8\%). Los mayores de 6 años eran 91 (39,2\%). Había más varones que mujeres, 138 niños (59\%) frente a 94 niñas (40,5\%). Aunque la nacionalidad más frecuente era la española, 171 niños $(73,7 \%), 59$ niños eran inmigrantes o hijos de inmigrantes $(25,4 \%$ ) y 2 niñas procedentes de adopción internacional $(0,9 \%)$. Lo cual supone 173 niños viviendo en familias españolas (74,6\%).
Respecto a las categorías diagnósticas más frecuentemente encontradas, hay que destacar que el asma fue la más prevalente, pues suponía 193 casos $(83,2 \%)$, seguido de cardiopatías congénitas 20 (8,6\%), neumonía recurrente $8(3,4 \%)$ y diabetes mellitus $7(3 \%)$ (tabla II).

En 6 niños existía contraindicación para administrar la vacuna antigripal (2,6\%).

De los 232 niños candidatos a la vacuna, se comprobó que la habían recibido 100 en esa campaña (desde octubre al 31 de diciembre de 2007). La cobertura de vacunación antigripal global calculada

Tabla I. Características de los niños identificados de riesgo para la gripe

\begin{tabular}{llrr}
\hline & Parámetros & $\mathbf{N}$ & $\%$ \\
\hline \multirow{2}{*}{ Edad } & Menores de 6 años & 141 & 60,8 \\
& Mayores de 6 años & 91 & 39,2 \\
& Varón & 138 & 59,0 \\
\multirow{2}{*}{ Nacionalidad } & Mujer & 94 & 40,5 \\
& Española & 173 & 74,6 \\
& Inmigrantes & 59 & 25,4 \\
\hline & Total & $\mathbf{2 3 2}$ & $\mathbf{1 0 0 , 0}$ \\
\hline
\end{tabular}

Tabla II. Frecuencia y porcentaje de pacientes pertenecientes a cada categoría diagnóstica de riesgo

\begin{tabular}{lrr}
\hline Diagnósticos & $\mathrm{N}$ & $\%$ \\
\hline Asma & 193 & 83,2 \\
Neumonía recurrente & 8 & 3,4 \\
Otra neumopatía & 2 & 0,9 \\
Cardiopatía congénita & 20 & 8,6 \\
Diabetes & 7 & 3,0 \\
Enfermedad neuromuscular & 1 & 0,4 \\
Artritis idiopática juvenil & 1 & 0,4 \\
\hline Total & $\mathbf{2 3 2}$ & $\mathbf{1 0 0 , 0}$ \\
\hline
\end{tabular}


Figura 2. Representación del recuento de pacientes vacunados de gripe y no vacunados según la categoría diagnóstica.

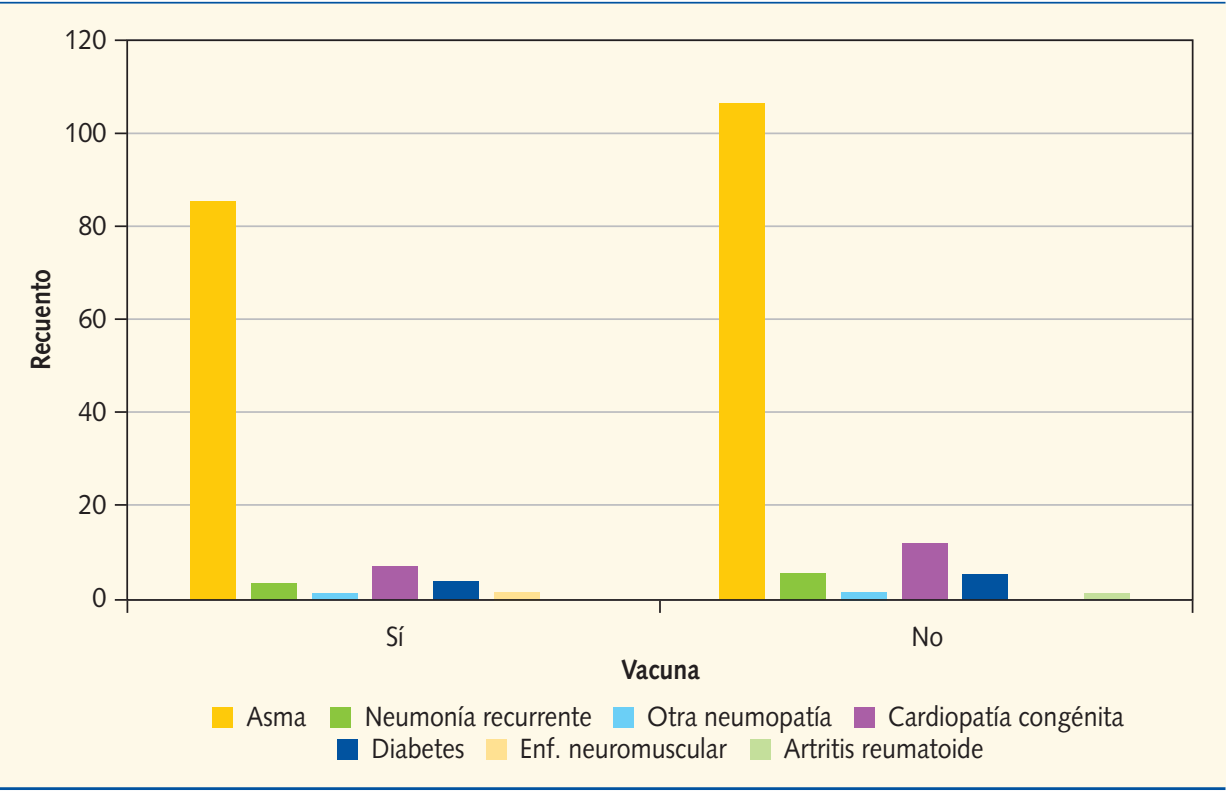

en niños de riesgo es $43,1 \%$ (intervalo de confianza [IC] del 95\%: 38-50).

De los 100 niños vacunados, 98 (98\%) recibieron una pauta completa y $2(2 \%)$ incompleta, pues habían recibido solo una dosis cuando por su edad y estatus inmunitario les correspondía recibir dos.

El recuento de vacunación para cada tipo de patología de base puede observarse en la figura 2 y en la tabla III. La cober-

Tabla III. Cobertura vacunal en cada grupo según la patología de base y en total

\begin{tabular}{lrrcc}
\hline Condición específica & N & $\%$ & $\begin{array}{c}\text { Niños } \\
\text { vacunados (N) }\end{array}$ & $\begin{array}{c}\text { Cobertura } \\
\text { vacunal (\%) }\end{array}$ \\
\hline Asma & 193 & 82,2 & 85 & 44,0 \\
Neumonía recurrente & 8 & 3,4 & 3 & 37,5 \\
Otra neumopatía & 2 & 0,9 & 1 & 50,0 \\
Cardiopatía congénita & 20 & 8,6 & 7 & 35,0 \\
Diabetes & 7 & 3,0 & 3 & 42,85 \\
Enfermedad neuromuscular & 1 & 0,4 & 0 & 0,0 \\
Artritis idiopática juvenil & 1 & 0,4 & 0 & 0,0 \\
\hline Total de niños con patología de base & 232 & 100 & 100 & 43,1 \\
\hline
\end{tabular}


tura vacunal para las condiciones más prevalentes fue $44 \%$ en caso de asma, $35 \%$ en cardiopatía congénita, $37,5 \%$ en neumonía recurrente y $42,85 \%$ en diabetes mellitus.

En las tablas IV, $\mathrm{V}$ y VI se muestran las coberturas vacunales en función de la edad, sexo y nacionalidad.

Se observa una mayor proporción de niñas vacunadas que de niños (odds ratio [OR]: 1,11; IC 95\%: 0,65-1,89). La proporción de niños menores de 6 años vacunados también es mayor que la de los de 6 a 14 años (OR: 1,59; IC 95\%: $0,92-2,73)$. La razón de prevalencia para la vacunación en los niños de familia española y niños de familia inmigrante es a favor de la vacunación en las familias es- pañolas (OR: 1,52; IC 95\%: 0,82-2,82) siendo $22 \%$ la fracción atribuible al hecho de ser vacunado por vivir en una familia española.

No hemos encontrado relación entre la edad, sexo y nacionalidad con el hecho de estar vacunado. La determinación de chi cuadrado para el estudio de relación entre cobertura vacunal y edad da una cifra de 2,85 ( $p=0,1-0,05)$. Al intentar estudiar la dependencia entre cobertura vacunal y sexo obtenemos chi cuadrado 0,15 ( $p>0,5)$ y entre cobertura vacunal y nacionalidad, el chi cuadrado es 1,81 ( $p=0,2-0-15)$. Por lo que podemos decir con un nivel de confianza del $95 \%$ que estas variables no son dependientes.

\begin{tabular}{|c|c|c|c|c|c|}
\hline Condición específica & $\mathrm{N}$ & $\%$ & Niños vacunados (N) & Cobertura vacunal (\%) & IC $95 \%$ \\
\hline$\leqslant 6$ años & 141 & 60,8 & 67 & 47,5 & $41,2-57,7$ \\
\hline$>6$ años & 91 & 39,2 & 33 & 36,2 & $28,3-47,8$ \\
\hline
\end{tabular}

\begin{tabular}{lccccc}
\hline Tabla V. Cobertura vacunal según el sexo & & & \\
\hline Condición específica & $\mathbf{N}$ & $\%$ & Niños vacunados (N) & Cobertura vacunal (\%) & IC 95\% \\
\hline Varones & 138 & 59,0 & 58 & 42,0 & $35-51,9$ \\
Mujeres & 94 & 40,5 & 42 & 44,7 & $36,9-56,9$ \\
\hline
\end{tabular}

\begin{tabular}{lccccc}
\hline \multicolumn{7}{l}{ Tabla Vl. Cobertura vacunal según la nacionalidad } \\
\hline Condición específica & $\mathbf{N}$ & $\%$ & Niños vacunados (N) & Cobertura vacunal (\%) & IC 95\% \\
\hline Español & 173 & 74,6 & 79 & 45,7 & $40,1-54,6$ \\
Inmigrante & 59 & 25,4 & 21 & 35,6 & $25,6-49,9$ \\
\hline
\end{tabular}




\section{Discusión}

Nuestro trabajo muestra que un $43,1 \%$ de los niños con alguna de las condiciones médicas más relevantes que suponen un riesgo para la gripe y sus complicaciones recibió la vacuna antigripal en la estación 2007-2008 en un centro de Atención Primaria. Son más vacunadas las niñas que los niños, los menores de 6 años y los que viven en familias españolas que los inmigrantes.

Hasta donde llega nuestro conocimiento, no existen estudios realizados en España de cobertura vacunal frente a la gripe realizados a esta población y en este medio. El poder realizar este tipo de trabajos exige tener censada a la población diana, en este caso los niños con condiciones médicas de base, lo cual solo es posible por parte de los profesionales que atienden directamente a estos pacientes.

En España los adultos con enfermedades crónicas, de edad inferior a 65 años y superior a 14 , recibieron la VA en un porcentaje de $26,6 \%$ en el año $2006^{11}$. Mientras, un $62,3 \%$ de adultos de más de 65 años la recibió en la campaña 2007-2008 ${ }^{12}$. Podemos intuir, que en general, pocos niños con condiciones de riesgo son vacunados frente a la gripe. En el caso de nuestros pacientes una cobertura de $43,1 \%$ es más elevada que los índices comunicados tanto en nuestro país, como en Europa o América.

Los trabajos españoles que estudian una aproximación al conocimiento de la cobertura de VA en niños con condiciones de riesgo apuntan que en el año 2004 , solo un $23,5 \%$ de los niños con EC ingresados en un hospital terciario habían sido vacunados frente a la gri$\mathrm{pe}^{13}$. Mientras, según un estudio poblacional, el 19,1\% de niños con condiciones médicas de base recibieron la VA en el 2006 en España ${ }^{11}$ y un 19,9\% de los niños con enfermedades respiratorias crónicas la recibían en el $2003^{14}$. En este caso los padres o tutores respondieron a un cuestionario, tanto para referir si existía alguna enfermedad crónica como para señalar si los niños habían recibido la vacunación. Nuestro estudio tiene la ventaja de que los datos no fueron proporcionados por un cuestionario, sino que se obtuvo del estudio de los historiales clínicos de los pacientes del centro de Atención Primaria.

En España disponemos de datos de cobertura vacunal frente a las vacunaciones sistemáticas gracias al inventario de estadísticas del Ministerio de Sanidad y Política Social' ${ }^{12}$. Para series básicas de primovacunación, el porcentaje de niños que recibe estas series es muy elevado, superior al $96 \%$, cifra que contrasta con las 
aportadas para el caso de la VA en niños de grupos de riesgo.

Los estudios en otros países aportan resultados que indican que la cobertura de VA es más baja de lo deseado. Así, en Italia se apunta una cobertura de $5,1 \%$ en el año 2000-2001 que se incrementa a $26,3 \%$ en el 2002-2003, en niños de riesgo, de edad inferior a 14 años, estudiados en un departamento de urgencias. También aporta el dato de encontrar 274 niños de riesgo de 5.286 estudiados $(5,1 \%)^{15}$. En Israel, un estudio indica que durante el invierno 2003-2004, solo un $6,5 \%$ de niños con EC fueron vacunados frente a la gripe ${ }^{16}$. En EE. UU. se objetiva una cobertura del $11,74 \%$ en el año 2002-2003 en niños de 1-17 años ${ }^{17}$, para adolescentes se describen cifras que oscilan del 8,3\% al 15,4\% en distintos años, la última cifra es en el año $2001^{18}$.

Las condiciones médicas descritas en la infancia que sientan la vacunación antigripal suelen estar presentes en un 7,4$14,2 \%$ de los niños ${ }^{1}$, destacando el asma que se describe como la condición más prevalente, suponiendo para algunos autores entre un $87,4-89,5 \%{ }^{18,19}$. En nuestro trabajo hemos encontrado un porcentaje discretamente inferior de niños con $\mathrm{EC}$, un $6,7 \%$, si bien es superior a lo descrito por Esposito et al. en Italia ${ }^{15}$, que señalábamos era de un 5,1\%. El asma la presentan un $83,2 \%$ de nuestros niños con EC, porcentaje similar al referido anteriormente. No debemos olvidar que nosotros hemos omitido deliberadamente a los niños con inmunosupresión por respetar la confidencialidad de algunos de estos procesos, creemos que este hecho no desvirtúa los resultados, pues en las series estos pacientes no superan el $1 \%$ de los niños con $\mathrm{EC}^{19}$.

El asma, por ser la EC más presente en la infancia, merece especial atención. Nuestros pacientes asmáticos tienen una cobertura de VA del $44 \%$, (IC 95\%: $38 \%-52,5 \%$ ) cifra muy satisfactoria. En Francia se ha comunicado una cobertura del $15,7 \%$ en niños asmáticos de edad 6-17 años en el año 2006$2007^{20}$. En EE. UU. se ha descrito una cobertura para el niño asmático del 9 $10 \%$ en campañas anteriores ${ }^{21}$, que en la actualidad se sitúan en un $29 \%{ }^{22}$. Es muy interesante destacar que el uso de herramientas de educación al niño asmático puede incrementar estas coberturas, pudiendo pasar de niveles del $13,1 \%$ al $59,4 \%$ tras realizar estrategias específicas ${ }^{23-25}$.

Un aspecto muy destacado de nuestro estudio es el hecho de explorar si existen desigualdades en salud en la atención al niño inmigrante con EC. La cobertura de vacuna antigripal en niños con EC fue 
mayor en los que viven en familias españolas que en los inmigrantes (OR: 1,52; IC 95\%: 0,82-2,82), sin que hayamos podido establecer una relación significativa entre estas variables. Esta faceta ha sido estudiada en adultos del área metropolitana de Madrid, encontrándose que sobre 7.341 sujetos había un $12,4 \%$ de inmigrantes. La cobertura de VA fue inferior en inmigrantes que en autóctonos $(11,2 \%$ vs. $25,9 \%)$. Estos autores, como en nuestro caso, tampoco encuentran significación a esta asociación ${ }^{26}$.

Tal vez una de las dificultades para realizar correctamente la vacunación de estos niños con EC sea su identificación. Algunos autores proponen listados de los códigos del CIE-9 asociados a un incremento del riesgo de complicaciones de la gripe $^{18,19}$. Los procesos que se identifican más frecuentemente con este sistema son: asma (87,4\%), displasia broncopulmonar $(1,7 \%)$, cardiopatía congénita $(1,9 \%)$, neoplasia maligna (1\%) y diabetes $(0,5 \%)$.

La $V A$ en nuestro medio es proporcionada por el Sistema Nacional de Salud a los niños de riesgo, libre de coste y con fácil accesibilidad a través de los centros de Atención Primaria; por lo que consideramos no existen razones económicas para que nuestros niños con EC no sean vacunados. Cuando se analiza el porqué de esta baja cobertura en niños de riesgo, la mayor parte de los autores apunta que una falta de recomendación por parte de los profesionales de Atención Primaria que atienden al niño se asocia significativamente con el fallo en recibir la $\mathrm{VA}^{27}$.

Queremos destacar que nuestro estudio pone de manifiesto una necesidad de salud, probablemente muy extendida, que requiere esfuerzos dirigidos a poder conseguir el objetivo recomendado por la OMS de alcanzar una cobertura de VA de un $75 \%$ en sujetos de riesgo en el $2010^{28}$.

\section{Agradecimientos}

A los siguientes profesionales del Centro de Salud Delicias Sur de Zaragoza: Francisca González Rubio, médico de familia; por su valiosa ayuda en la explotación de datos del sistema OMI. A las pediatras: M. ${ }^{a}$ Jesús Cabañas, Amparo Fuertes y $M{ }^{a}$ Teresa Solans; por habernos permitido trabajar con la base de datos de sus pacientes. A las enfermeras de Pediatría: M. ${ }^{a}$ Fe Crespo, Concepción Esteban y Asun Verón, por su dedicación a la vacunación. 


\section{Bibliografía}

1. Erhart LM, Rangel MC, Lu PJ, Singleton JA. Prevalence and characteristics of children at increased risk for complications from influenza, United States, 2000. J Pediatr. 2004;144:191-5.

2. Niranjan B, Wright JG, Broder KR, Murray EL, Greenberg ME, Glover MJ, et al. Influenza-associated deaths among children in the United States, 2003-2004. N EngI J Med. 2005;353:2559-67.

3. Neuzil KM, Wright PF, Mitchel EF, Griffin $M R$. The burden of influenza illness in children with asthma and other chronic medical conditions. J Pediatr. 2000;137:856-64.

4. Reichert TA, Sugaya N, Fedson DS, Glezen WP, Simonsen L, Tashiro M. The Japanese Experience with Vaccinating Schoolchildren against Influenza. N Engl J Med. 2001;344:889-96.

5. Piedra PA, Gaglani MJ, Kozinetz CA, Herschler $G$, Riggs $M$, Griffith $M$, et al. Herd immunity in adults against influenza-related illnesses with use of the trivalent-live attenuated influenza vaccine (CAIV-T) in children. Vaccine. 2005;23:1540-8.

6. Glezen WP. Universal influenza vaccination and live attenuated influenza vaccination of children. Pediatr Infect Dis J. 2008;27(10):s104-s109.

7. Fiore $A E$, Shay DK, Broder K, Iskander JK, Uyeki TM, Mootrey G, et al. Centers for Disease Control and Prevention (CDC); Advisory Committee on Immunization Practices (ACIP). Prevencion and control of influenza: recommendations of the Advisory Committee on Immunization Practices (ACIP), 2008. MMWR Recomm Rep. 2008;57 (RR-7):1-60.

8. Childhood Vaccination Schedule. Finland. EUVAC.NET [consultado el 01/08/2009]. Disponible en www.euvac.net/graphics/euvac/vaccina tion/finland.html

9. Bernaola Iturbe E, Giménez Sánchez F, Baca Cots M, De Juan Martín F, Díez Domingo J, Garcés
Sánchez M, y cols. Comité Asesor de Vacunas de la Asociación Española de Pediatría, Madrid, España. Calendario vacunal de la Asociación Española de Pediatría: Recomendaciones 2009. An Pediatr (Barc). 2009;70:72-82.

10. Comité Asesor de Vacunas de la Asociación Española de Pediatría. Calendario de vacunación de la Asociación Española de Pediatría: recomendaciones 2007. An Pediatr (Barc). 2007;66:62-9.

11. Jiménez-García $R$, Hernández-Barrera $V$, Carrasco-Garrido $P$, López de Andrés A, Pérez $N$, de Miguel AG. Influenza vaccination coverages among children, adults, health care workers and immigrants in Spain: related factors and trebds, 2003-2006. J Infect. 2008;57:472-80.

12. Coberturas de vacunación datos estadísticos. Ministerio de Sanidad y Política Social. España [consultado el 18/08/2009]. Disponible en www. msc.es/profesionales/saludPublica/prevPromo cion/vacunaciones/coberturas.htm

13. Sánchez Callejas A, Campins Martí M, Martínez Gómez X, Pinós Tella L, Hermosilla Pérez $E_{1}$ Vaqué Rafart J. Influenza vaccination in patients admitted to a tertiary hospital. Factors associated with coverage. An Pediatr (Barc). 2006;65:331-6.

14. López de Andrés $A$, Carrasco-Garrido $P$, Hernández-Barrera $V$, de Miguel $A G$, JiménezGarcía R. Coverages and factors associated with influenza vaccination among subjects with chronic respiratory diseases in Spain. Eur J Public Health. 2008;18(2):173-7.

15. Esposito S, Marchisio P, Droghetti R, Lambertini L, Faelli N, Bosis $S$, et al. Influenza vaccination coverage among children with hifh-risk medical conditions. Vaccine. 2006;24:5251-5.

16. Stein M, Yossepovitch O, Somekh E. Influenza vaccine coverage in paediatric population from central Israel. J Infect. 2005;50:382-5.

17. Cho BH, Kolasa MS, Messonier ML. Influenza vaccination coverage rate among high-risk chil- 
dren during the 2002-2003 influenza season. Am J Infect Control. 2008;36:582-7.

18. Nakamura MM, Lee GM. Influenza vaccination in adolescents with high-risk conditions. Pediatrics. 2008;122:920-8.

19. Daley MF, Barrow J, Pearson K, Crane LA, Gao $D$, Stevenson $J M$, et al. Identification and recall of children with chronic medical conditions for influenza vaccination. Pediatrics. 2004;113:e2633.

20. Rancé $F$, Chave $C$, de Blic J, Deschildre $A$, Donato $L$, Dubus JC, et al. Influenza vaccination coverage in asthmatic children in France in 20062007. Arch Pediatr. 2008;15:1724-8.

21. Kramarz P, DeStefano F, Gargiullo PM, Davis $\mathrm{RL}$, Chen RT, Mullooly JP, et al. Influenza vaccination in children with asthma in Health Maintenance Organizations. Vaccine. 2000;18:2288-94.

22. Centers for Disease Control and Prevention (CDC). Influenza vaccination coverage among children with asthma. United States, 2004-05 influenza season. MMWR Morb Mortal Wkly Rep. 2007;56(9):193-6.

23. Martin E. Improving influenza vaccination rates for pediatrics asthmatics by use of asthma educational tool and patient electronic care system. Clin Pediatr (Phila). 2008;47:588-92.
24. Esposito S, Pelucci C, Tel F, Chiarelli G, Sabatini $C$, Semino $M$, et al. Factors conditioning effectiveness of a reminder/recall system to improve influenza vaccination in asthmatic children. Vaccine. 2009;29;27:6233-5.

25. Britto MT, Schoettker PJ, Pandzik GM, Weiland J, Mandel KE. Improving influenza immunisation for high-risk children and adolescents. Qual Saf Health Care. 2007;16:363-8.

26. Jiménez-García $R$, Hernández-Barrera $V$, Carrasco-Garrido $P$, de Andrés Al, Esteban Peña MM, de Miguel AG. Coverage and predictors of influenza vaccination among adults living in a large metropolitan area in Spain: a comparison between the immigrant and indigenous populations. Vaccine. 2008;26:4218-23.

27. Mirza A, Subedar A, Fowler SL, Murray DL, Arnold S, Tristam D, et al. Influenza vaccine: awareness and barriers to immunization in families of children with chronic medical conditions other than asthma. South Med J. 2008;101:1101-5.

28. World Health Organization. WHO recommends influenza vaccine composition for Northern Hemisphere 2005-2006 influenza season [consultado el 21/08/2009]. Disponible en www.who. int/mediacentre/news/notes/2005/np05/en/in dex.html 